\title{
Ab initio calculations for the square-lattice anisotropic Heisenberg model
}

\author{
Document Version \\ Accepted author manuscript
}

Link to publication record in Manchester Research Explorer

\section{Citation for published version (APA):}

Bishop, RF., \& Farnell, DJJ. (1998). Ab initio calculations for the square-lattice anisotropic Heisenberg model. In D. Neilson, \& RF. Bishop (Eds.), Recent Progress in Many-Body Theories: Proceedings of the 9th International Conference, Sydney, Australia, 21-25 July 1997 (pp. 433-442). (Series on Advances in Quantum Many-Body Theory; Vol. 1). World Scientific Publishing Co. Pte. Ltd.

http://personalpages.manchester.ac.uk/staff/raymond.bishop/RFB_papers/[142] SerAQMBT_1(1998)433

\section{Published in:}

Recent Progress in Many-Body Theories

\section{Citing this paper}

Please note that where the full-text provided on Manchester Research Explorer is the Author Accepted Manuscript or Proof version this may differ from the final Published version. If citing, it is advised that you check and use the publisher's definitive version.

\section{General rights}

Copyright and moral rights for the publications made accessible in the Research Explorer are retained by the authors and/or other copyright owners and it is a condition of accessing publications that users recognise and abide by the legal requirements associated with these rights.

\section{Takedown policy}

If you believe that this document breaches copyright please refer to the University of Manchester's Takedown Procedures [http://man.ac.uk/04Y6Bo] or contact uml.scholarlycommunications@manchester.ac.uk providing relevant details, so we can investigate your claim.

\section{OPEN ACCESS}




\section{Series on Advances in Quantum Many-Body Theory - Vol. 1}

The Proceedings of the 9th International Conference

\section{RECENT PROGRESS IN MANY-BODY THEORIES}

Sydney, Australia $\quad$ July $21-25,1997$

Editors

\section{David Neilson}

The University of New South Wales, Sydney, Australia

\section{Raymond F. Bishop}

UMIST, Manchester, UK 


\section{Published by}

World Scientific Publishing Co. Pte. Ltd.

P O Box 128, Farrer Road, Singapore 912805

USA office: Suite 1B, 1060 Main Street, River Edge, NJ 07661

UK office: 57 Shelton Street, Covent Garden, London WC2H 9HE

British Library Cataloguing-in-Publication Data

A catalogue record for this book is available from the British Library.

\section{RECENT PROGRESS IN MANY-BODY THEORIES}

Copyright $\odot 1998$ by World Scientific Publishing Co. Pte. Ltd.

All rights reserved. This book, or parts thereof, may not be reproduced in any form or by any means, electronic or mechanical, including photocopying, recording or any information storage and retrieval system now known or to be invented, without written permission from the Publisher.

For photocopying of material in this volume, please pay a copying fee through the Copyright Clearance Center, Inc., 222 Rosewood Drive, Danvers, MA 01923, USA. In this case permission to photocopy is not required from the publisher.

ISBN 981-02-3369-8

Printed in Singapore by Uto-Print 


\title{
AB INITIO CALCULATIONS FOR THE SQUARE-LATTICE ANISOTROPIC HEISENBERG MODEL
}

\author{
R.F. BISHOP and D.J.J. FARNELL \\ Department of Physics, UMIST \\ (University of Manchester Institute of Science and Technology) \\ P.O. Box 88, Manchester M60 1QD, UK
}

\begin{abstract}
Interest in lattice quantum spin systems as models of quantum magnets has increased with the discovery of new and interesting magnetic materials. Here we use a well-known technique of quantum many-body theory, namely the coupled-cluster method (CCM), to investigate the nearest-neighbour, spin- $\frac{1}{2}$, anisotropic Heisenberg model on the square lattice. Ground-state expectation values for quantities such as the ground-state energy and the sublattice magnetisation are determined to an accuracy comparable with that of the best of other available techniques including Monte Carlo methods. In order to demonstrate this point we present results for various values of the anisotropy parameter, including those for the isotropic Heisenberg model and the isotropic $X Y$ model. We show that it is now possible to determine the presence and position of the quantum phase transitions using $a b$ initio CCM calculations, and furthermore that we can accurately predict the critical behaviour at these points.
\end{abstract}

\section{Introduction}

The coupled cluster method (CCM) [1-5] is a widely-used technique of quantum many-body theory. It has been applied over the last five or so years to many lattice Hamiltonian systems with considerable success. These systems include various spin lattices problems [6-9] of much current interest. As well as being models of quantum magnets, quantum spin lattices often exhibit quantum phase transitions and complicated phase diagrams. The strong effects of quantum mechanics on these systems means that they can contain interesting and novel ground and excited states which have no classical counterparts.

In this article we present a specific application of the CCM to the spin- $\frac{1}{2}$ anisotropic Heisenberg (or $X X Z$ ) model on the two-dimensional (2D) square lattice. In the next section, we present the CCM formalism in general terms before specializing to its application to the $2 \mathrm{D} X X Z$ model in Sec. 3. In Sec. 4 we describe our results for the ground-state expectation values of the energy and sublattice magnetisation, as well as results for the critical behaviour of this model. Finally, in Sec. 5 we briefly discuss the relevance, quality and possible extensions of our results.

\section{The CCM for Quantum Spin Lattices}

The single-reference version of the normal variant of the CCM used here requires a suitable single model or reference state $|\Phi\rangle$, in terms of which a systematic description of the many-body (i.e., in the present case, multi-spin) correlations or fluctuations may be given. We defer till later the important question of how to choose $|\Phi\rangle$ in practice, but note now that it is required only to be a cyclic vector with respect to two well-defined Abelian subalgebras of multi-configurational creation operators 
$\left\{C_{I}^{+}\right\}$and their Hermitian-adjoint destruction counterparts $\left\{C_{I}^{-} \equiv\left(C_{I}^{+}\right)^{\dagger}\right\}$. Thus, $|\Phi\rangle$ plays the role of a vacuum state with respect to a suitable set of (mutually commuting) many-body creation operators $\left\{C_{I}^{+}\right\}$,

$$
C_{I}^{-}|\Phi\rangle=0, \quad \forall I \neq 0,
$$

with $C_{0}^{-} \equiv I$, the identity operator. These operators are also complete in the many-body Hilbert (or Fock) space,

$$
I=|\Phi\rangle\left\langle\Phi\left|+\sum_{I \neq 0} C_{I}^{+}\right| \Phi\right\rangle\langle\Phi| C_{I}^{-} .
$$

The choice of the operators $\left\{C_{I}^{+}\right\}$depends on the choice of $|\Phi\rangle$, but for spinlattice problems $C_{I}^{+}$will generally involve products of the basic $S U(2)$ spin operators $\left\{s_{k}^{+}, s_{k}^{-}, s_{k}^{z}\right\}$ on different lattice sites $k$, which obey the fundamental commutation relations,

$$
\left[s_{k}^{z}, s_{l}^{ \pm}\right]= \pm s_{k}^{ \pm} \delta_{k l} \quad ; \quad\left[s_{k}^{+}, s_{l}^{-}\right]=2 s_{k}^{z} \delta_{k l},
$$

where $s_{k}^{ \pm} \equiv s_{k}^{x} \pm \mathrm{i} s_{k}^{y}$. The set-index $I$ will thus generally incorporate the indices for a set of lattice sites. We discuss particular choices of $\left\{|\Phi\rangle, C_{I}^{+}\right\}$in more detail below in the context of a specific example.

The exact ground-state energy eigenket and eigenbra vectors, $|\Psi\rangle$ and $\langle\tilde{\Psi}|$ respectively, of a many-body system described by a Hamiltonian $H$,

$$
H|\Psi\rangle=E_{g}|\Psi\rangle ; \quad\langle\tilde{\Psi}| H=E_{g}\langle\tilde{\Psi}|,
$$

are now specified within the single-reference normal CCM as follows,

$$
\begin{gathered}
|\Psi\rangle=e^{S}|\Phi\rangle ; \quad S=\sum_{I \neq 0} \mathcal{S}_{I} C_{I}^{+} ; \\
\langle\tilde{\Psi}|=\langle\Phi| \tilde{S} e^{-S} ; \quad \tilde{S}=1+\sum_{I \neq 0} \tilde{\mathcal{S}}_{I} C_{I}^{-} .
\end{gathered}
$$

The correlation operator $S$ is thus decomposed entirely in terms of the multiconfigurational creation operators $\left\{C_{I}^{+}\right\}$, and similarly for $\tilde{\mathcal{S}}$ in terms of the destruction operators $\left\{C_{I}^{-}\right\}$. Although the manifest Hermiticity, $(\langle\tilde{\Psi}|)^{\dagger}=|\Psi\rangle /\langle\Psi \mid \Psi\rangle$, is lost, the intermediate normalization condition, $\langle\tilde{\Psi} \mid \Psi\rangle=\langle\Phi \mid \Psi\rangle=\langle\Phi \mid \Phi\rangle \equiv 1$ is imposed. The full set of coefficients $\left\{\mathcal{S}_{I}, \tilde{\mathcal{S}}_{I}\right\}$ now provides a complete CCM description of the many-body ground state. For example, an arbitrary operator $A$ has a ground-state expectation value,

$$
\bar{A} \equiv\langle\tilde{\Psi}|A| \Psi\rangle=\left\langle\Phi\left|\tilde{S} e^{-S} A e^{S}\right| \Phi\right\rangle=\bar{A}\left(\left\{\mathcal{S}_{I}, \tilde{\mathcal{S}}_{I}\right\}\right)
$$

The exponentiated form of the eigenket parametrization of Eq. (5) ensures the proper counting of the independent fluctuations of excited multi-spin configurations (described by the set-index $I$ ) with respect to $|\Phi\rangle$, which are present in the exact ground state $|\Psi\rangle$, and the exact incorporation of the linked cluster theorem of Goldstone. The latter guarantees the size-extensivity of all relevant physical quantities, 
and allows us to work directly in the thermodynamic limit, $N \rightarrow \infty$, where $N$ is the number of lattice spins.

By taking appropriate projections of the ground-state ket and bra Schrödinger equations (i.e., with states $\langle\Phi| C_{I}^{-}$and $C_{I}^{+}|\Phi\rangle$, respectively), we obtain coupled sets of equations which may be solved to obtain the coefficients $\left\{\mathcal{S}_{I}\right\}$ and $\left\{\tilde{\mathcal{S}}_{I}\right\}$. Completely equivalently, the correlation coefficients $\left\{\mathcal{S}_{I}, \tilde{\mathcal{S}}_{I}\right\}$ may be determined variationally by requiring that the ground-state energy functional $\bar{H}\left(\left\{\mathcal{S}_{I}, \tilde{\mathcal{S}}_{I}\right\}\right)$, defined as in Eq. (7), is stationary with respect to variations in each of the (independent) variables of the full set. The following coupled sets of equations are thereby easily derived,

$$
\begin{gathered}
\delta \bar{H} / \delta \tilde{S}_{I}=0 \Rightarrow\left\langle\Phi\left|C_{I}^{-} e^{-S} H e^{S}\right| \Phi\right\rangle=0, \quad \forall I \neq 0 ; \\
\delta \bar{H} / \delta \mathcal{S}_{I}=0 \Rightarrow\left\langle\Phi\left|\tilde{S} e^{-S}\left[H, C_{I}^{+}\right] e^{S}\right| \Phi\right\rangle=0, \quad \forall I \neq 0 .
\end{gathered}
$$

Equation (8) ensures that the ground-state energy at the stationary point has the simple form

$$
E_{g}=E_{g}\left(\left\{\mathcal{S}_{I}\right\}\right)=\left\langle\Phi\left|e^{-S} H e^{S}\right| \Phi\right\rangle,
$$

which also follows simply by projecting the ground-state ket equation (4) with $\langle\Phi| e^{-S}$. This bi-variational formulation does not, however, lead to an upper bound for $E_{g}$ when the summations over configurations $\{I\}$ in Eqs. (5) and (6) for $S$ and $\tilde{S}$ are truncated in specific approximations, since the exact Hermiticity between $|\Psi\rangle$ and $\langle\tilde{\Psi}|$ will thereby be lost. On the other hand, the Hellmann-Feynman theorem is preserved in all such approximations.

Equation (8) clearly represents a coupled set of nonlinear multinomial equations for the $c$-number correlation coefficients $\left\{\mathcal{S}_{I}\right\}$. The well-known nested commutator expansion for the similarity-transformed Hamiltonian,

$$
\hat{H} \equiv e^{-S} H e^{S}=H+[H, S]+\frac{1}{2 !}[[H, S], S]+\cdots,
$$

taken together with the fact that all of the individual components of $S$ in the sum in Eq. (5) commute with one another, imply that each element of $S$ in Eq. (5) is thus directly linked to the Hamiltonian in each of the non-vanishing terms in Eq. (11). Each of the coupled set of equations (8) is hence of linked cluster type. What is more, each of these equations is also of finite length when expanded using Eq. (11), since this otherwise infinite series will actually terminate at a finite order here, provided only that each term in the Hamiltonian $H$ contains a finite number of single-site spin operators, as is usually the case. The CCM parametrization thus leads in a very natural way to a workable scheme, which can also be efficiently implemented as described in more detail below.

We turn now to the choice of $|\Phi\rangle$ and the operators $\left\{C_{I}^{+}\right\}$for spin-lattice problems. To be specific we henceforth restrict ourselves to spin- $\frac{1}{2}$ quantum antiferromagnets on bipartite lattices, in regions where the corresponding classical limit is described by a generalized Néel-like ordering in which all spins on each sublattice are separately aligned in the coordinates of a global spin quantization axis and corresponding global spin axes. In such cases it is a simple matter (and see Sec. 3 for 
specific details in the case considered here) to introduce a different local quantization axis and spin coordinates on each sublattice, by a suitable rotation in spin space, so that the corresponding Néel-like state becomes a fully aligned ("ferromagnetic") state in the local axes. This "ferromagnetic" state is chosen as the uncorrelated CCM model state, $|\Phi\rangle$, in which all spins point, say, along the respective negative $z$-axis of the corresponding local frames,

We also need to consider approximation schemes whereby the expansions of $S$ and $\tilde{S}$ in Eqs. (5) and (6) may be truncated to some finite or infinite subset of the full set of independent (fundamental) multi-spin configurations. The three most commonly employed schemes have been: (1) the SUBn scheme, in which all correlations involving only $n$ or fewer spins are retained, but no further restriction is made concerning their spatial separation on the lattice; (2) the SUB $n-m$ subapproximation, in which all $\mathrm{SUB} n$ correlations spanning a range of no more than $m$ adjacent lattice sites are retained; and (3) the localized LSUB $m$ scheme, which retains all multi-spin correlations over all possible distinct locales on the lattice defined by $m$ or fewer contiguous sites. For the results reported below we adopt only the LSUB $m$ scheme here.

\section{The Spin- $\frac{1}{2} X X Z$ Antiferromagnet on the 2D Square Lattice}

The $X X Z$ Hamiltonian is specified in terms of global spin coordinates as follows,

$$
H=\sum_{\langle i, j\rangle}\left[s_{i}^{x} s_{j}^{x}+s_{i}^{y} s_{j}^{y}+\Delta s_{i}^{z} s_{j}^{z}\right],
$$

where the sum on $\langle i, j\rangle$ counts all nearest-neighbour pairs once. On the 2D square lattice this model has no exact solution, unlike its 1D chain counterpart which is exactly integrable using the Bethe ansatz. The $X X Z$ model appears to have at least three distinct regimes: an Ising-like phase for sufficiently large values of the anisotropy parameter $\Delta$, which is characterized by nonzero Néel order wherein nearest-neighbour spins in the ground-state wave function align antiparallel along the $z$-axis; a planar-like phase in which the spins in the ground-state wave function are believed to lie in the $x y$ plane; and a ferromagnetic phase. Note that at $\Delta=0$ we recover the isotropic case of the $X Y$ model.

For $\Delta>1$ the classical Hamiltonian of Eq. (12) on the 2D square lattice (and, indeed, on any bipartite lattice) is minimized by a perfectly antiferromagnetically Néel-ordered state in the $z$-direction, whereas for $-1<\Delta<1$ it is minimized by a correspondingly ordered state with spins antiferromagnetically aligned along any direction in the $x y$ plane, say along the $x$-axis. Thus, we see that even for the same spin model and lattice, different choices of model state may be preferable, depending on the particular regime of parameter space in which we are interested. For present purposes we shall utilize both of these classical Néel states, namely the $z$-aligned Néel state and the $x$-aligned Néel state (with the latter henceforth denoted as the planar model state), for two separate sets of corresponding CCM calculations. In both cases we now set up different local sets of spin axes on both sublattices so that in the local coordinates all spins in both model states point in the negative $z$-direction, as discussed above in Sec. 2. 
For the $z$-aligned Néel state we simply perform a rotation of the axes of the uppointing spins by $180^{\circ}$ about the $y$-axis, such that: $s^{x} \rightarrow-s^{x}, s^{y} \rightarrow s^{y}, s^{z} \rightarrow-s^{z}$. The Hamiltonian of Eq. (12) may then be written in these local axes as

$$
H^{z}=-\frac{1}{2} \sum_{\langle i, j\rangle}\left[s_{i}^{+} s_{j}^{+}+s_{i}^{-} s_{j}^{-}+2 \Delta s_{i}^{z} s_{j}^{z}\right],
$$

where the superscript $z$ on $H^{z}$ reminds us that the Hamiltonian is written in the local spin coordinate axes appropriate to the $z$-aligned Néel model state.

In order to produce a "ferromagnetic" model state for the planar model state in the local frames, we rotate the axes of the left-pointing spins (i.e., those pointing in the negative $x$-direction) in the planar state by $90^{\circ}$ about the $y$-axis, and the axes of the corresponding right-pointing spins by $-90^{\circ}$ about the $y$-axis. (Note that the positive $z$-axis is defined here to point upwards and the positive $x$-axis is defined to point rightwards.) Thus, the transformations of the local axes are described such that: $s^{x} \rightarrow s^{z}, s^{y} \rightarrow s^{y}, s^{z} \rightarrow-s^{x}$, for the left-pointing spins; and such that: $s^{x} \rightarrow-s^{z}, s^{y} \rightarrow s^{y}, s^{z} \rightarrow s^{x}$, for the right-pointing spins. The transformed Hamiltonian of Eq. (12) may now be written in these local axes as

$$
H^{p}=-\frac{1}{4} \sum_{\langle i, j\rangle}\left[(\Delta+1)\left(s_{i}^{+} s_{j}^{+}+s_{i}^{-} s_{j}^{-}\right)+(\Delta-1)\left(s_{i}^{+} s_{j}^{-}+s_{i}^{-} s_{j}^{+}\right)+4 s_{i}^{z} s_{j}^{z}\right]
$$

where, again, the superscript $p$ on $H^{p}$ reminds us that the Hamiltonian is written in the local spin coordinate axes appropriate to the planar model state. It is important to recall that since the Hamiltonians $H, H^{z}$, and $H^{p}$ of Eqs. (12)-(14) differ only by similarity transformations their eigenvalue spectra are identical.

We now note that the CCM is especially suited to the evaluation of the ket- and bra-state equations by computer-algebraic techniques, and a detailed description of how this may be efficiently achieved is given in Ref. [8]. Once the bra and ket states are known at a particular approximation level then the approximate ground-state energies and other ground-state expectation values may be obtained. In the next section we describe our results for this model using both model states.

\section{Results}

\subsection{Ground-State Energy}

Results for the ground-state energy using the two model states are illustrated in Fig. 1 at the LSUB4 and LSUB6 levels of approximation, where they are compared with the Monte Carlo results of Barnes et al. [10]. The results for the isotropic Heisenberg model (which are identical using both model states for this case) are summarized in Table 1 for calculations using both model states as CCM reference states. We also present results for the isotropic $X Y$ model using the planar model state.

In order to compare our results with those from other methods, we attempt a simple heuristic extrapolation of our LSUB $m$ results to the limit $m \rightarrow \infty$ at the isotropic Heisenberg point $(\Delta=1)$. As has already been found elsewhere [7], 
Table 1: Results obtained for the spin- $\frac{1}{2} X X Z$ model on the 2D square lattice using CCM LSUBm approximations $(m=2,4,6,8)$. $N_{F}^{p}$ denotes the number of fundamental configurations for the planar model state, and $N_{F}^{z}$ denotes the number of fundamental configurations for the $z$-axis Néel model state. The ground-state energy per spin, $E_{g} / N$, and the sublattice magnetisation, $M^{+}$, at the isotropic Heisenberg point $(\Delta=1)$ and at the isotropic $X Y$ point $(\Delta=0)$ are shown, as well as extrapolated results in the limit $m \rightarrow \infty$. Various critical anisotropy parameters are also given. $\Delta_{F}^{p}$ and $\Delta_{A}^{p}$ indicate the LSUBm critical points for the planar model state corresponding to the ferromagnetic and antiferromagnetic phase transitions. $\Delta_{A}^{z}$ indicates the critical point for the $z$-axis Néel model state corresponding to the antiferromagnetic phase transition. Note that there are no terminating points in the LSUB2 approximation. (Brackets indicate the error in the last significant figure shown.)

\begin{tabular}{|c|c|c|c|c|c|c|c|c|c|}
\hline \multirow[t]{2}{*}{$m$} & \multirow[t]{2}{*}{$N_{F}^{P}$} & \multirow[t]{2}{*}{$N_{F}^{z}$} & $E_{g} / N$ & $M^{+}$ & $E_{g} / N$ & $\bar{M}^{+}$ & \multirow[t]{2}{*}{$\Delta_{F}^{p}$} & \multirow[t]{2}{*}{$\Delta_{A}^{p}$} & \multirow[t]{2}{*}{$\Delta_{A}^{z}$} \\
\hline & & & \multicolumn{2}{|l|}{$\Delta=1$} & \multicolumn{2}{|c|}{$\Delta=0$} & & & \\
\hline 2 & 1 & 1 & -0.64833 & 0.841 & -0.54031 & 0.950 & - & - & - \\
\hline 4 & 10 & 7 & -0.66366 & 0.764 & -0.54727 & 0.916 & -1.250 & 1.648 & 0.577 \\
\hline 6 & 131 & 75 & -0.66700 & 0.727 & -0.54833 & 0.901 & -1.084 & 1.286 & 0.7631 \\
\hline 8 & 2793 & 1287 & -0.66817 & 0.705 & -0.54862 & 0.894 & $?$ & $?$ & 0.8429 \\
\hline$\infty$ & - & - & -0.66968 & 0.62 & -0.54892 & 0.869 & -0.95 & 1.00 & $0.96(4)$ \\
\hline
\end{tabular}

our results seem to extrapolate well to their asymptotic value with a leading $m$ dependent correction that scales as $\mathrm{m}^{-2}$. In this way we obtain an extrapolated value for the ground-state energy per spin of $E_{g} / N=-0.66968$. This compares very well with the best Monte Carlo simulation value [11] of $-0.66934 \pm 0.00004$, and is very much more accurate, by comparison with this Monte Carlo value, than the linear spin-wave theory (LSWT) result [12] of $E_{g} / N=-0.658$. At the isotropic $X Y$ point $(\Delta=0)$, using the planar model state $[8,9]$, we obtain an extrapolated value for the energy of -0.54892 , which may be compared to a result of series expansion calculations [13] of $-\mathbf{0 . 5 4 8 8}$.

Figure 1 illustrates that at a given LSUBm level of approximation the CCM result for the ground-state energy using the $z$-aligned Néel model state lies lower than its counterpart using the planar model state for $\Delta>1$, and vice versa for $\Delta<1$. This result is precisely as would be expected classically, and it illustrates the power of being able to employ different CCM model states which are specifically geared to different possible phases. If we were simply to take that solution with the lower energy for each value of $\Delta$ (for which we note, however, that there is no real justification), we would infer that there is a phase transition at $\Delta=1$ between a phase with Ising-like order at $\Delta>1$ and a planar-like phase for $\Delta<1$.

We note, furthermore, and much more importantly, that each separate calculation also yields evidence of such a phase transition. Thus, we find that beyond certain critical values, $\Delta_{c}$, of the anisotropy parameter there exists no physically reasonable solution to the LSUB $m$ CCM equations for $m \geq 4$, as is illustrated for the cases $m=4,6$ in Fig. 1. In previous work [7] we have related this characteristic breakdown of the CCM equations at certain critical points to actual phase transitions of the real system, and we explore this further in Sec. 4.2 below. A useful means to detect phase transitions within the LSUBm scheme is to calculate the 


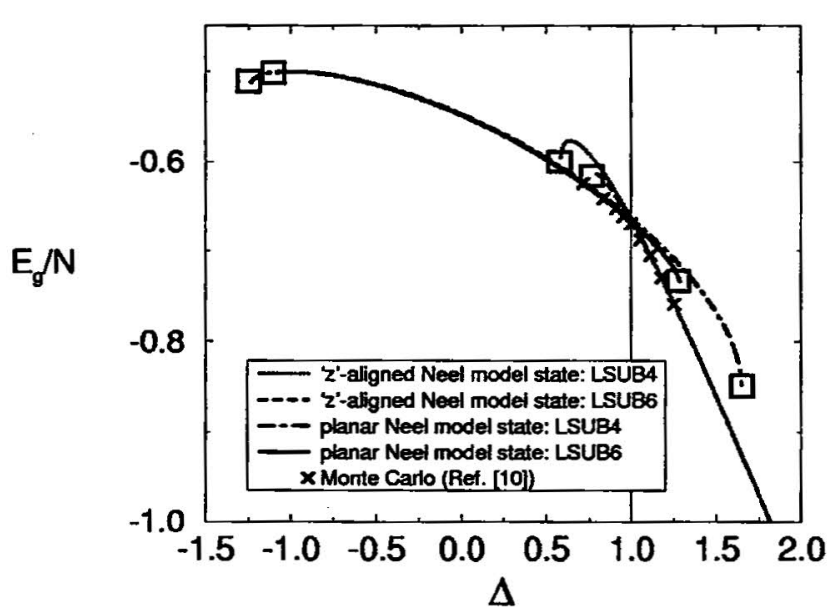

Figure 1: Results for the CCM gxound-state energy of the spin- $\frac{1}{2} X X Z$ model on the 2D square lattice, using the LSUBm approximation with $m=4,6$ based on both the planar and $z$-aligned Néel model states, compared to the Monte Carlo results of Ref. [10]. LSUBm critical points, $\Delta_{F}^{p}, \Delta_{A}^{p}$ and $\Delta_{A}^{z}$, are indicated by the boxes.

so-called anisotropy susceptibility, $\chi_{a}$,

$$
\chi_{a} \equiv-\frac{\partial^{2}\left(E_{g} / N\right)}{\partial \Delta^{2}},
$$

Since the CCM equations are known analytically, $\chi_{a}$ and all other derivatives may also be calculated directly (i.e., from analytic equations). We find that $\chi_{a}$ diverges at the critical points.

More specifically, we find that for the CCM calculations based on the planar model state $\chi_{a}$ diverges at critical values $\Delta_{c}=\Delta_{F}^{p}$ and $\Delta_{A}^{p}$, corresponding to the ferromagnetic and antiferromagnetic phase transitions respectively, for all LSUB $m$ approximations with $m>2$. These results are illustrated in Table 1, which also displays the single critical point at $\Delta_{c}=\Delta_{A}^{z}$ for the CCM calculations based on the $z$-aligned Néel model state, and which again corresponds to the antiferromagnetic phase transition. As one might hope, the position of the critical point $\Delta_{F}^{p}$ becomes closer to the true ferromagnetic phase transition at $\Delta=-1$ as the approximation level is increased. Also, both $\Delta_{A}^{p}$ and $\Delta_{A}^{z}$ appear to converge with increasing LSUB $m$ index $m$, and for a given value of $m$ always bound the point $\Delta=1$ at which the true antiferromagnetic phase transition is believed (by symmetry arguments) to lie. We have shown elsewhere [7] how the corresponding SUB2-m results for $\Delta_{A}^{z}$ seem to approach the full SUB2 value as $m^{-2}$, and the same rule fitted to the LSUB $m$ results yields the corresponding predictions for the extrapolated antiferromagnetic point indicated in Table 1 , namely $\Delta_{A}^{z} \approx 0.96 \pm 0.04$, and $\Delta_{A}^{p} \approx 1.00$. Both predictions are compatible with each other and with the expected value $\Delta_{A}=1$. 


\subsection{Sublattice Magnetization}

We now introduce the sublattice magnetization, which characterises the degree of quantum order inherent in the CCM wave functions. By inserting the CCM parametrizations of Eqs. (5) and (6) we find,

$$
M^{+} \equiv-\frac{2}{N} \sum_{k=1}^{N}\left\langle\tilde{\Psi}\left|s_{k}^{z}\right| \Psi\right\rangle=-\frac{2}{N} \sum_{k=1}^{N}\left\langle\Phi\left|\tilde{S} e^{-S} s_{k}^{z} e^{S}\right| \Phi\right\rangle,
$$

where $s_{k}^{z}$ is in the local coordinates of each sublattice. Evaluation of the sublattice magnetization requires both the ket- and bra-state cluster correlation coefficients. The actual procedure to do this is straightforward, and is also described in more detail elsewhere [8].

Table 1 summarizes the results for the sublattice magnetization at the isotropic Heisenberg point, $\Delta=1$, and at the isotropic $X Y$ point, $\Delta=0$. We note again that the corresponding LSUB $m$ results for $M^{+}$using both model states at a given truncation level $m$ are identical at $\Delta=1$. We find that over the entire range $-1<\Delta<1$ our results seem to converge well to a nonzero in-plane long-range order, with a nonzero planar sublattice magnetization, whereas for all $\Delta>1$ we have comparable nonzero long-range order along the spin $z$-axis and a nonzero $z$ component of sublattice magnetization. The fact that there is also a divergence in $M^{+}$near the critical points $\Delta_{A}^{z}$ and $\Delta_{A}^{p}$ strongly reinforces our interpretation of these points as reflecting a phase transition.

We again attempt a simple heuristic extrapolation of our LSUBm results for $M^{+}$ at the Heisenberg point $(\Delta=1)$ to the limit $m \rightarrow \infty$, in order to compare our results with those from other calculations. As has been found elsewhere [7], the results for $M^{+}$extrapolate well to their asymptotic value with a leading correction that scales as $m^{-1}$. As shown in Table 1 we thus obtain an extrapolated value, $M^{+}=0.62$. This compares extremely well with the best available Monte Carlo simulation value of Runge [11], $M^{+}=0.615 \pm 0.005$, and with the value $M^{+}=0.62 \pm 0.02$ from series expansion techniques [14]. At the isotropic $X Y$ point we obtain a similarly extrapolated value of $M^{+}=0.869$, which again compares very well with the result of series expansion calculations [13] of $M^{+}=0.872$.

\subsection{Critical Properties}

We have demonstrated that the CCM calculations provide an accurate representation of the ground-state properties of the spin- $-\frac{1}{2}$ square-lattice $X X Z$ model. We now use our CCM formalism to predict the critical behaviour of the physical observables near such phase transitions, i.e., that we can extract from the LSUB $m$ results useful information on critical indices.

Thus, the critical index for the singular (non-analytic) term in $E_{g} / N$ near an LSUB $m$ critical point $\Delta_{c}(m)$ can first be obtained, for example, by direct examination of the anisotropy susceptibility, $\chi_{a} \equiv-\partial^{2}\left(E_{g} / N\right) / \partial \Delta^{2}$, of Eq. (15). For $m>2$ we find,

$$
\chi_{a}^{m}(\Delta) \longrightarrow \bar{\chi}_{a}^{m}\left|\Delta-\Delta_{c}(m)\right|^{-\alpha_{0}} ; \quad \Delta \rightarrow \Delta_{c}(m) .
$$


Direct calculation for the LSUBm approximations using both the $z$-aligned and planar Néel model states shows that for $m>2$ we have $\alpha_{0} \approx 1.500 \pm 0.005$. However, the prefactors $\bar{\chi}_{a}^{m}$ in Eq. (17) are themselves strongly dependent on the truncation index $m$. We may now use a variant of the so-called coherent anomaly method (CAM) of Suzuki [15] to extract further information. Thus, we attempt to fit $\bar{\chi}_{\boldsymbol{a}}^{m}$ with the coherent anomaly form,

$$
\bar{\chi}_{a}^{m} \longrightarrow K\left|\Delta_{c}(\infty)-\Delta_{c}(m)\right|^{\nu} ; \Delta \rightarrow \Delta_{c}(\infty),
$$

where $K$ is a constant. Thus, as explained by Suzuki [15], one may intuit or prove that the exact $\chi_{a}(\Delta)$ has the critical form,

$$
\chi_{a}(\Delta) \rightarrow \kappa\left|\Delta-\Delta_{c}(\infty)\right|^{-\alpha_{0}+\nu} ; \Delta \rightarrow \Delta_{c}(\infty) \equiv \Delta_{c},
$$

where $\kappa$ is a constant.

A CAM analysis along these lines of the LSUB $m$ results based on the $z$-aligned Néel state gives $\nu \approx 1.25$ using the $\Delta_{A}^{z}(4)$ and $\Delta_{A}^{z}(6)$ data, and $\nu \approx 0.97$ using the $\Delta_{A}^{z}(6)$ and $\Delta_{A}^{z}(8)$ data. We thus obtain a singular term in $E_{g} / N$ near $\Delta_{A}^{z}$ with a critical exponent $2-\alpha_{0}+\nu \approx 1.50-1.75$. This may be compared with the corresponding value of $3 / 2$ for both the mean-field-like CCM SUB2 approximation (in which all 2-spin-flip correlation terms are retained, however far apart on the lattice) and linear spin-wave theory (LSWT). A corresponding analysis may also be carried out on the LSUBm results based on the planar model state near $\Delta_{A}^{p}$. We again find $\alpha_{0} \approx 1.500 \pm 0.005$ for both the LSUB4 and LSUB6 results, and a corresponding critical anomaly based on these of $\nu \approx 1.27$. We thus obtain a singular term in $E_{g} / N$ near $\Delta_{A}^{p}$ with a critical exponent $2-\alpha_{0}+\nu \approx 1.77$. These preliminary data are clearly compatible with the hypothesis that the critical exponent in the energy is the same on both sides of the antiferromagnetic phase transition $\Delta_{A}$.

Similar CAM analyses can also be performed for the ground-state energy near the corresponding ferromagnetic critical point $\Delta_{F}^{p}$, and for such other properties as the sublattice magnetization $M^{+}$near any of these critical points.

\section{Conclusions}

We have shown in this article that it is possible to model accurately the properties of the spin- $\frac{1}{2} X X Z$ model on the square lattice in two regions of the phase space using the CCM. We have produced results for the ground-state energy and sublattice magnetisation which are in full agreement with the best of the alternative approximate theories, including quantum Monte Carlo techniques.

It has also been shown that the critical points, which mark the phase boundaries of this model, are predicted to good accuracy, and that we can now make quantitative statements regarding the criticality at these points. The CCM is therefore essentially unique at present in the field of many-body theory in its ability to predict with high accuracy both ground-state expectation values and the critical behaviour of the system in the vicinity of quantum phase transitions between states of different quantum order.

An interesting and fundamental extension of the CCM treatment of the quantum spin lattice problems presented here would be to describe their behaviour at 
non-zero temperatures. A possible method of achieving this would be to extend the formalism by utilizing the general framework of thermofield dynamics [16]. We note also that Mukherjee [17] and others have suggested alternative ways to extend the CCM to incorporate a thermal averaging procedure.

Finally, we note that the CCM techniques discussed here may also be applied with equal ease and success to such frustrated spin lattice problems as the Heisenberg model on a triangular lattice [8]. By contrast we note that such frustrated lattices are much more difficult to deal with by quantum Monte Carlo methods than bipartite lattices of the type considered here.

\section{Acknowledgements}

We thank J.B. Parkinson, Y. Xian, and C. Zeng for useful discussions. One of us (RFB) also gratefully acknowledges a research grant from the Engineering and Physical Sciences Research Council (EPSRC) of Great Britain.

\section{References}

1. F. Coester, Nucl Phys. 7, 421 (1958); F. Coester and H. Kümmel, ibid. 17, 477 (1960).

2. J. Čižek, J. Chem. Phys. 45, 4256 (1966); Adv. Chem. Phys. 14, 35 (1969).

3. R.F. Bishop and K.H. Lührmann, Phys. Rev. B 17, 3757 (1978).

4. R.F. Bishop and H. Kümmel, Phys. Today 40(3), 52 (1987).

5. R.F. Bishop, Theor. Chim. Acta 80, 95 (1991).

6. R.F. Bishop, J.B. Parkinson, and Yang Xian, (a) Phys. Rev. B 43, 13782 (1991); (b) ibid. 44, 9425 (1991); (c) ibid. 46, 880 (1992); (d) Theor. Chim. Acta 80, 181 (1991); (e) J. Phys.: Condens. Matter 4, 5783 (1992); (f) ibid. 5, 9169 (1993).

7. R.F. Bishop, R.G. Hale, and Y. Xian, Phys. Rev. Lett. 73, 3157 (1994).

8. C. Zeng, D.J.J. Farnell, and R.F. Bishop, UMIST preprint UMIST/Phys/TP/97-2 (1997).

9. R.F. Bishop, D.J.J. Farnell, and J.B. Parkinson, J. Phys.: Condens. Matter 8, 11153 (1996); D.J.J. Farnell, S.E. Krüger, and J.B. Parkinson, ibid. 9, 7601 (1997).

10. T. Barnes, D. Kotchan, and E.S. Swanson, Phys. Rev. B 39, 4357 (1989).

11. K.J. Runge, Phys. Rev. B 45, 12292 (1992); ibid. 45, 7229 (1992).

12. P.W. Anderson, Phys. Rev. 86, 694 (1952); T. Oguchi, ibid. 117, 117 (1960).

13. C.J. Hamer, J. Oitmaa and W. Zheng, Phys. Rev. B 43, 10789 (1991).

14. R.R.P. Singh, Phys. Rev. B 39, 9760 (1989); R.R.P. Singh and D.A. Huse, ibid. 40, 7247 (1989); W. Zheng, J. Oitmaa, and C.J. Hamer, ibid. 43, 8321 (1991).

15. M. Suzuki, J. Phys. Soc. Japan 55, 4205 (1986).

16. H. Umezawa, H. Matsumoto, and M. Tachiki, Thermo Field Dynamics and Condensed States, 'North-Holland, Amsterdam, 1982; H. Umezawa and Y. Yamanaka, Adv. Phys. 37, 531 (1988).

17. G. Sanyal, S.H. Mandal, and D. Mukherjee, Chem. Phys. Lett. 192, 55 (1992). 